\title{
Back to the edge: relative coordinate system for use-wear analysis
}

\author{
Ivan Calandra ${ }^{1}$ (1) $\cdot$ Lisa Schunk ${ }^{1,2}$ - Alice Rodriguez ${ }^{3}$. Walter Gneisinger ${ }^{1} \cdot$ Antonella Pedergnana $^{1} \cdot$ Eduardo Paixao $^{1,4}$. \\ Telmo Pereira ${ }^{4} \cdot$ Radu lovita ${ }^{3,5} \cdot$ Joao Marreiros ${ }^{1,2,4}$
}

Received: 8 November 2018 / Accepted: 28 January 2019 / Published online: 11 November 2019

(C) The Author(s) 2019

\begin{abstract}
Use-wear studies rely heavily on experiments and reference collections to infer the function of archeological artifacts. Sequential experiments, in particular, are necessary to understand how use-wear develops. Consequently, it is crucial to analyze the same location on the tool's surface during the course of an experiment. Being able to relocate the area of interest on a sample is also essential for reproducibility in use-wear studies. However, visual relocation has limited applicability and there is currently no easy and efficient alternative. Here we propose a simple protocol to create a coordinate system directly on the sample. Three ceramic beads that serve as reference markers are adhered onto the sample, either with epoxy resin or acrylic polymer. The former is easier to work with but the latter is reversible so it can be applied to archeological samples too. The microscope's software then relocates the position(s) of interest. We demonstrate the feasibility of this approach and measure its repeatability by imaging the same position on an experimental flint blade 10 times with two confocal microscopes. Our results show that the position can be relocated automatically with a horizontal positional repeatability of approximately $14 \%$ of the field of view. Quantitative surface texture measurements according to ISO 25178 vary due to this positional inaccuracy, but it is still unknown whether this variation would mask functional differences. Although still perfectible, we argue that this protocol represents an important step toward repeatability and reproducibility in experimental archeology, especially in use-wear studies.
\end{abstract}

Keywords Confocal microscopy $\cdot$ ISO $25178 \cdot$ Lithics $\cdot$ Repeatability $\cdot$ Surface texture $\cdot$ Traceology

\section{Introduction}

Experimental archeology has become one of the most important tools in Paleolithic archeology. In use-wear studies in particular, experiments are necessary to build up reference collections to which the archeological samples are compared. Yet, most studies have focused only on the wear produced after the experiment, while neglecting the state before use in the experiment (e.g., Bofill et al. 2013; Portillo et al. 2013; Stemp et al. 2015; Sano and Oba 2015; Key et al. 2015; Watson and Gleason 2016; Galimova and Sitdikov 2017; Queffelec et al. 2018). However, no surface is perfectly smooth, meaning that the topography already present before the experiment (i.e., surface topography due to tool manufacturing or "unused" topography) has an effect on the development of wear. To tackle this problem, some studies
Electronic supplementary material The online version of this article (https://doi.org/10.1007/s12520-019-00801-y) contains supplementary material, which is available to authorized users.

Ivan Calandra

calandra@rgzm.de

1 TraCEr, Laboratory for Traceology and Controlled Experiments at MONREPOS Archaeological Research Centre and Museum for Human Behavioural Evolution, RGZM, Neuwied, Germany

2 Institute for Prehistoric and Protohistoric Archaeology, Johannes Gutenberg University, Mainz, Germany
3 Anthrotopography Laboratory, Center for the Study of Human Origins, Department of Anthropology, New York University, New York City, NY, USA

4 ICArEHB, Interdisciplinary Center for Archaeology and Evolution Human Behaviour, University of Algarve, Faro, Portugal

5 Early Prehistory and Quaternary Ecology, University of Tübingen, Tübingen, Germany 
have analyzed nearby unused areas (e.g., Stemp et al. 2013, 2018) or unused experimental samples (e.g., Stemp and Stemp 2001, 2003; Evans and Donahue 2005, 2008; Evans and Macdonald 2011; Macdonald 2014; Werner 2018) that are assumed to show the same unused topography. However, no two surfaces are identical: even two surfaces from the same sample will have different unused topographies. This difference becomes fundamental when quantitative 3D use-wear analyses are applied. Other studies (e.g., Asryan et al. 2014; Ollé and Vergès 2014; Evans et al. 2014; Pedergnana et al. 2016; Chabot et al. 2017; Benito-Calvo et al. 2018; Martisius et al. 2018) have therefore analyzed how use-wear develops through sequential experiments, in which the researchers tried to visually relocate the same surface before, during, and after the experiment. However, this approach only works if the surface has not changed to the point that it is not recognizable anymore (Martisius et al. 2018). Moreover, it is highly dependent on the scale of analysis, where smaller areas are harder to find again visually than larger ones. This is true, for example, when using scanning electron (SEM) or confocal microscopes at high magnifications. In summary, there is a need to automatically relocate the area of interest without visual comparisons (Martisius et al. 2018). To our knowledge, only Stemp and Stemp (2003) and Martisius et al. (2018) developed such a system by scratching the sample to create a reference point on the sample.

Here we present a simple protocol to automatically relocate a position on a sample, either before, during, and after an experiment, or for the re-analysis of samples with different microscopes/analyses (e.g., archeological samples, samples stored in reference collections). The method described here can work with any type of microscope featuring a motorized stage and associated software. In order to quantify the potential of the method to affect the measurement of surface topography, we applied it with confocal microscopes. While we tested this method only on experimental samples, it can also be applied to archeological samples.

The goals of the present study were to (1) demonstrate the coordinate system functionality on two confocal microscopes from different manufacturers, (2) measure the X-Y positional repeatability of the coordinate system functionality on each microscope, and (3) measure the variability in surface topography due to this positional (in)accuracy. We were therefore not interested in comparing the positional repeatability of each microscope. We argue that, whatever the repeatability of the microscope and associated software used is, the coordinate system functionality available to the user should be applied because it is in any case better than finding the location manually and visually.

Hereafter, following Leach (2013), the term surface topography will be used to describe the overall surface structure, while surface form is defined as the shape of the object, and surface texture is what remains when the form is removed from the topography. These definitions differ from Evans et al. (2014), where texture describes the roughness and topography the waviness (both included in Leach's (2013) texture), the distinction between roughness and waviness being based on wavelength filters (see below).

\section{Materials and methods}

\section{Samples}

We selected two experimental tools displaying use-wear. Both tools (FLT1-4 and FLT1-7) are blades knapped from flint from the French Pyrenees (Narbonne-Sigean Basin). They were used in mechanical bi-directional linear (cutting-like) action on dry wood (Pinus sp.) boards. Each blade performed 250 strokes of $2 \times 30 \mathrm{~cm}$ at $0.5 \mathrm{~m} . \mathrm{s}^{-1}$ with a $4.5 \mathrm{~kg}$ load applied onto the tool (Pereira et al. in prep.).

Ceramic beads were adhered onto the ventral side of the samples to be used as reference points for the definition of a coordinate system directly on the samples. For this, epoxy resin was used on sample FLT1-7. Epoxy resin is, in the strict meaning of the term, not reversible and should not be applied to archeological samples. Therefore, Paraloid B72, an acrylic polymer used routinely in archeological conservation, was also tested and applied to sample FLT1-4. Paraloid B72 is easily reversible (i.e., dissolved) with acetone or ethyl acetate. The sample FLT1-4 was used to demonstrate the applicability of Paraloid B72 (see below for details); it was not used for further analysis, as the aim was to show the feasibility of the procedure using a conservation grade adhesive. It should be remembered that the specific glass transition temperature $(\mathrm{Tg})$ of an adhesive might cause the adhesive to flow, if placed under a localized heat source such as microscope lighting other than LED.

\section{Cleaning procedure}

The samples were placed in new individual plastic bags filled with $\sim 300 \mathrm{~mL}$ of demineralized water and a nonionic detergent (BASF Plurafac LF901, $1 \mathrm{~g} / \mathrm{L}=1 \% w / v$; BASF SE, Ludwigshafen, Germany). They were then immersed into an ultrasonic bath (EMAG Emmi $20 \mathrm{HC}$ ) pre-heated at $40^{\circ} \mathrm{C}$ and left for 5-10 min to reach the bath temperature. Ultrasonic action was applied for $15 \mathrm{~min}$ at $45 \mathrm{KHz}$ and $150 \mathrm{~W}$.

Subsequently, samples were rinsed in two steps: (1) the bags were filled and emptied 3 times with tap water to remove surfactant residues and (2) the bags were filled with $\sim 300 \mathrm{~mL}$ demineralized water and immersed into the $40{ }^{\circ} \mathrm{C}$ bath for $5-10 \mathrm{~min}$ and another $15 \mathrm{~min}$ of ultrasonic action at $45 \mathrm{KHz}$ and $150 \mathrm{~W}$ to remove residues 
from all previous steps. Finally, the samples were covered and left to air-dry overnight.

The measured area (around the edge) was cleaned again with 2-propanol 70\% v/v and lens cleaning tissues just before acquisition.

\section{Beads}

Ceramic beads were adhered to the ventral side of the samples either with epoxy resin (FLT1-7) or Paraloid B72 (FLT1-4) (see Online Resource 1).

The epoxy resin component (Epoxydharz L) was mixed with the hardener component (Härter $S$ ) in a 10:4 ratio by weight. Black pigment concentrate (Universal-Farbpaste, Schwarz), maximum $2 \%$ weight, was added to make the preparation more visible. Thixotropic filler (Thixotropiermittel) was also added on demand to reduce viscosity. All components are manufactured by R\&G Faserverbundwerkstoffe GmbH (Waldenbruch, Germany). The pot life of the entire preparation is up to $1.5 \mathrm{~h}$, and the curing time is approximately $24 \mathrm{~h}$. Resin drops were applied via a needle-point onto the flint surface under a Leica M420 stereo-microscope at low magnification $(\times 120-\times 180)$. After about $30 \mathrm{~min}, 100-200 \mu \mathrm{m}$ diameter ceramic beads (SiLiBead Type ZY-S 0,1-0,2; Sigmund Lindner GmbH, Warmensteinach, Germany) were applied via a needlepoint wetted with 2-propanol, although we realized later that the residual resin was enough for the needle-point to remain sticky to handle the beads without alcohol (see Online Resource 1). This 30 min delay was necessary to ensure that the beads would not sink into the resin pool. The beads were centered as precisely as possible in the resin pool.

Paraloid B72, an EA/MMA copolymer, was dissolved in ethyl acetate (Kremer Pigmente GmbH \& Co. KG, Aichstetten, Germany) to $25 \% w / w$. The dissolved polymer starts to set by evaporation of solvent more or less instantaneously. Polymer drops were applied via a needle-point onto the flint surface under a binocular. Ceramic beads were applied via a needle-point wetted with ethyl acetate.

In both cases, the beads were adhered approximately in the same plane as the region of interest, several millimeters away from the active parts of the tool (e.g., edge). This ensures that the beads would not be removed during the experiments. Additionally, epoxy resin and ceramics are strong and resilient materials that should offer sufficient resistance to accidental displacement. The beads were adhered 5-10 $\mathrm{mm}$ apart; they should not be placed in a straight line but no specific angle between the beads is required (Fig. 1). While decreasing the size of the beads might offer processual advantages, they become increasingly challenging to manipulate.

\section{D data acquisition}

\section{Scanning conditions}

A mold was taken of the dorsal side of the flint blade (FLT1-7) to ensure that the sample will stay stable during acquisition (Fig. 2). Indeed, plasticine (Patafix) was found to be too soft so that the sample was moving a few micrometers per minute, which was enough to be detected and to influence the acquisition. Moreover, plasticine can be difficult to remove from (archeological) samples, which can compromise future residue analysis (e.g., Pedergnana et al. 2016). The mold was made of Provil novo Putty regular set (Kulzer GmbH, Hanau, Germany), a two-component silicone impression material comprising base paste and matching catalyst paste for addition curing that hardens in $3-5 \mathrm{~min}$ at ambient room conditions.

The sample was positioned with the measured area as horizontal as possible (Fig. 2) to minimize the vertical ( $z$ axis) measuring range. For this, the sample had to be tilted slightly. The tilt required is dependent on the morphology of the sample and on the area of interest on the sample. Temperature and humidity were measured constantly in both labs.

\section{Confocal microscopes and acquisition settings}

We acquired 3D surface topography data on sample FLT1-7 with two confocal microscopes, also called optical profilometers: (1) an upright light microscope Axio Imager.Z2 Vario coupled to a laser-scanning confocal microscope (LSCM) LSM 800 MAT (Carl Zeiss Microscopy $\mathrm{GmbH}$ ) at the laboratory for Traceology and Controlled Experiments (TraCEr) at MONREPOS, Germany, and (2) a Sensofar S neox optical profilometer (Sensofar Metrology, Barcelona, Spain) at the Anthrotopography Laboratory at New York University, USA. They are hereafter referred to as "LSM" and "S neox", respectively. Other confocal microscopes may feature a similar functionality, and, if so, could also be used; for example, the Olympus LEXT or the Leica DCM8 (identical to the S neox, except for the objectives).

All relevant information and settings are listed in Table 1. Both systems were turned on at least $1 \mathrm{~h}$ before starting acquisition, so that all components were warmed up to limit thermic drift during acquisition. The coordinate system was created and calibrated (see section "Coordinate system" below) with $10 \times$ objectives, while the $3 \mathrm{D}$ surface topography was acquired with $50 \times$ objectives. The fields of view are fixed for each microscope and objective combination. With the $50 \times$ objectives, the fields of view measure $255.56 \times 255.56 \mu \mathrm{m}$ on the LSM 

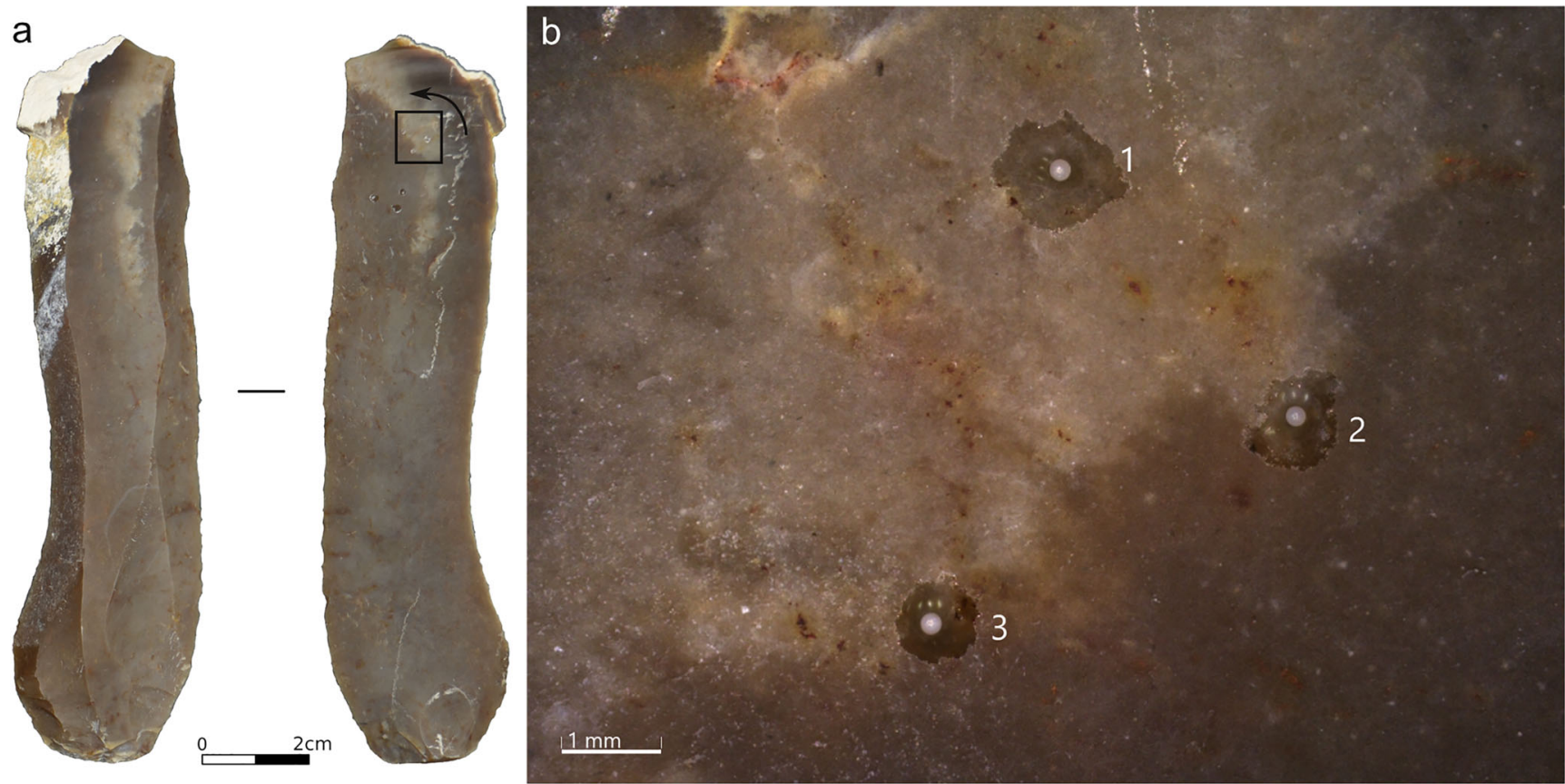

C

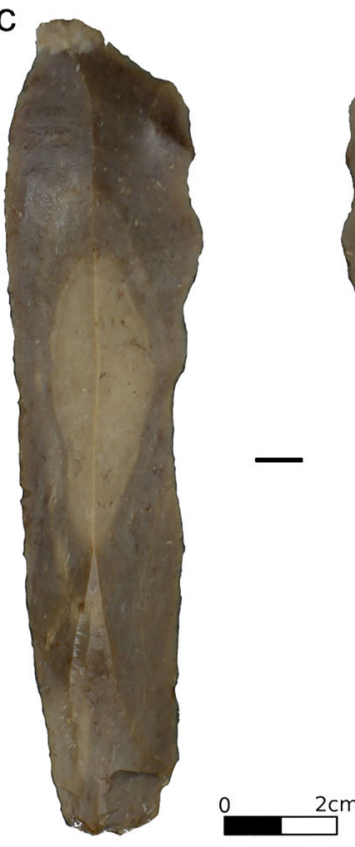

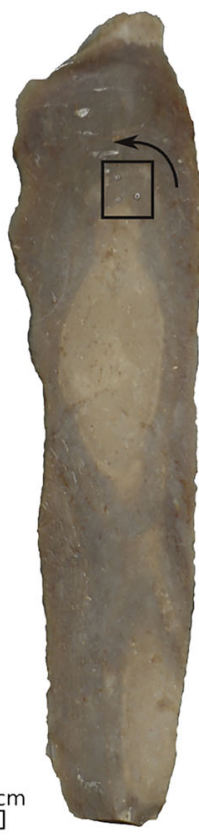

d

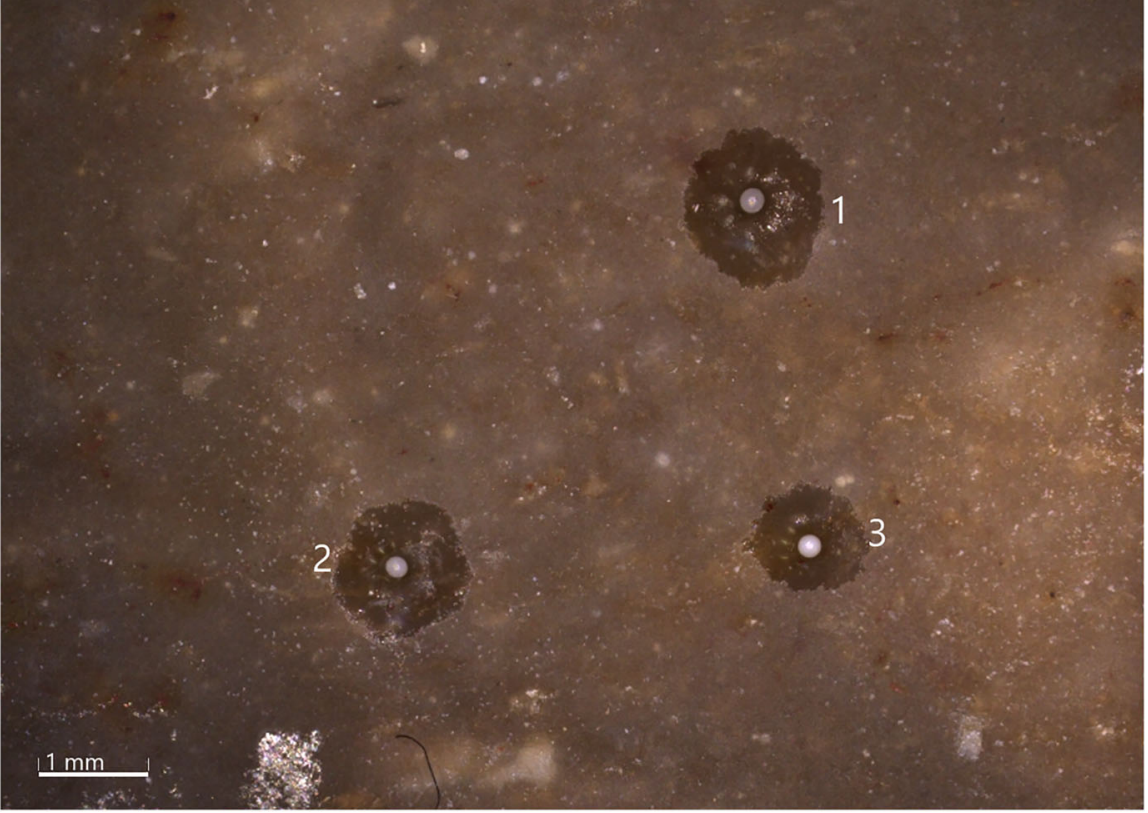

Fig. 1 Overview of the two samples prepared for this study, FLT1-4 (a-b) and FLT1-7 (c-d). Photos of the tools (a, c) and close-ups of the attached beads $(\mathbf{b}, \mathbf{d})$ on each sample, rotated $90^{\circ}$ counter clockwise relative to the overview photos. The numbering of the beads corresponds to the order of the reference markers. Photos were taken with a Nikon DSLR camera

and $350.88 \times 264.19 \mu \mathrm{m}$ on the $\mathrm{S}$ neox (Table 1 ). The frame size is fixed on the $S$ neox $(1360 \times 1024$ pixels $)$ but it can be adjusted with up to $6144 \times 6144$ pixels on the LSM. Frame size was therefore set to $991 \times 991$ pixels on the LSM in order for the pixel size (or measuring point spacing $=$ field of view/frame size) to be identical on both microscopes: $0.258 \mu \mathrm{m}$ in $X$ and $Y$ directions.
D610 with a Nikon AF-S VR Micro-Nikkor 105 mm f/2.8G IF-ED lens. Close-ups were acquired with a Smartzoom 5 equipped with a PlanApo $1.6 \times / 0.1$ objective at $\times 34$ total on-screen $\left(17.5^{\prime \prime}\right)$ magnification (Carl Zeiss Microscopy $\mathrm{GmbH}$ )

\section{Coordinate system}

Zeiss's ZEN blue (for the LSM) and Sensofar's SensoSCAN (for the $\mathrm{S}$ neox) software packages can achieve the same goal: automatically find a location on a sample again and again. Three markers on the sample are needed for both systems. These markers serve as reference points to define a coordinate 


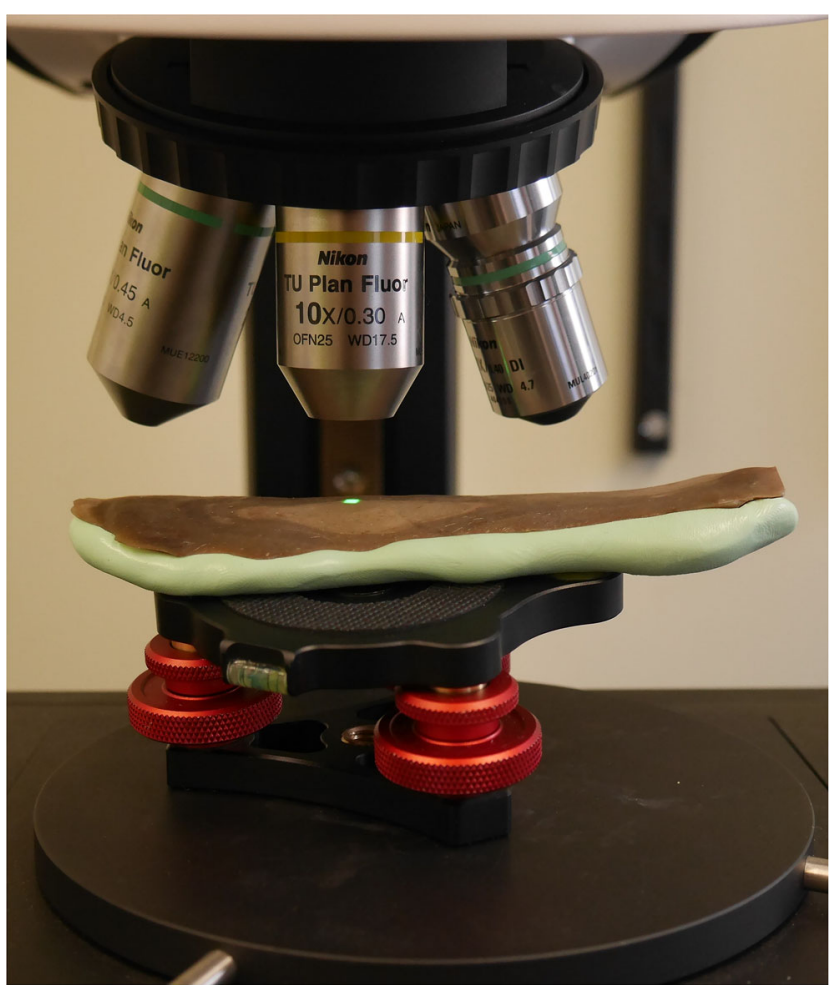

Fig. 2 View of the sample FLT1-7 during definition of the coordinate system with the S neox: a manual goniometer is used to orientate the region of interest as horizontally as possible and a silicon mold holds the sample in place. The same setup was used on the LSM

system directly on the sample (see Online Resource 2 for stepby-step instructions on each microscope's software).

Acquisition was performed on each microscope by applying the following procedure. (1) The coordinate system was created before the first scan, using the three adhered beads as reference points with the $10 \times$ objective. (2) One area with visible polish was located and scanned with the $50 \times$ objective. (3) The sample was removed from the microscope stage and lifted from its molded support. (4) The sample was repositioned on the stage with its orientation (rotation and tilt) as close as possible to the original orientation. (5) The coordinate system was then calibrated again with the $10 \times$ objective and the microscope software automatically moved the microscope XY stage so that the same location was positioned under the objective. (6) This location was scanned again with the $50 \times$ objective, without further manual adjustments of the stage. Deviations from scan to scan therefore only result from prepositioning (rotation and tilt) errors, positional reproducibility of the stage motors, and inaccuracy (rounding) of the software calculations. (7) The process was repeated so that the sample was scanned 10 times with each microscope.

Since the coordinate systems cannot be exchanged from one microscope and software package to the other, we had to create and use two different coordinate systems, one for each microscope. However, we tried to manually find the location of the first scan on the first microscope (LSM) for the first scan on the second microscope (S neox), so that the acquired surfaces are as similar as possible. Therefore, the first scan on each microscope was located manually; the subsequent nine scans were located automatically with the software packages. This is not critical because, as stated above, the goal is not to compare the two pieces of equipment.

\section{D data processing}

The resulting 3D surface data were processed in ConfoMap v7.4.8633 (a derivative of MountainsMap Imaging Topography developed by Digital Surf, Besançon, France). A template was applied to each 3D surface $(n=20)$ with the following procedure: (1) extract the topographic layer, (2) extract the $255.56 \times 255.56 \mu \mathrm{m}$ area $(991 \times 991$ pixels $)$ from the top left corner, (3) apply a Gaussian low-pass S-filter ( $\mathrm{S}_{1}$ nesting index $=0.425 \mu \mathrm{m}$, end effects managed) to remove noise and keep the primary surface, (4) apply an F operator (polynomial of degree 3 ) to remove the form and keep the SF surface, i.e., texture, (5) apply a Gaussian high-pass L-filter (L nesting index $=127 \mu \mathrm{m}$, end effects managed) to filter out the waviness and keep the SL surface, i.e., roughness, and (6) threshold the surface between 0.010 and $99.9 \%$ material ratio to remove the aberrant positive and negative spikes. Steps 1-2 were applied to surfaces from the S neox only. See Online Resource 3 for details and for results on each surface.

This template follows Digital Surf's Metrology Guide (accessible at https://guide.digitalsurf.com/en/guide.html) and therefore the ISO 25178 norm (International Organization for Standardization 2012) as closely as possible, but it should not be expected that lithic tool surfaces require the exact same processing as dictated by the ISO norms, defined for industrial applications. Moreover, such surfaces constrain some processing settings. We therefore adapted the cutoff values for the filters based on field of view as follows: The $\mathrm{L}$ nesting index cannot be larger than half the shortest side (breadth) of the field of view, i.e., $255.56 / 2=127.78 \mu \mathrm{m}$, truncated to $127 \mu \mathrm{m}$. The ISO 4287/4288 norms (International Organization for Standardization 1996, 1997) state that $\lambda_{\mathrm{s}}$ (2D equivalent of the $S_{1}$ nesting index) should be 300 times smaller than $\lambda_{c}$ (2D equivalent of the $\mathrm{L}$ nesting index), i.e., $0.426 \mu \mathrm{m}$, rounded to $0.425 \mu \mathrm{m}$. These norms also recommend the pixel size to be no larger than one-fifth of $\lambda_{\mathrm{s}} / \mathrm{S}_{1}$, i.e., $0.0852 \mu \mathrm{m}$, which would result in 255.56/0.0852 $=3000$ pixels in $X$ and in $Y$ directions on the LSM, or 350.88/0.0852 $=4119$ pixels in $X$ and 264.19/ $0.0852=3111$ pixels in $Y$ on the $\mathrm{S}$ neox. The detector of the LSM can measure up to $6144 \times 6144$ pixels, but the CCD camera sensor of the $\mathrm{S}$ neox is limited to $1360 \times 1024$ pixels. So pixel size was limited to $0.258 \mu \mathrm{m}$ in both $X$ and $Y$ directions, as explained above. 
Table 1 Acquisition settings on both confocal microscopes

\begin{tabular}{|c|c|c|c|}
\hline Setting & & LSM & S neox \\
\hline \multirow[t]{2}{*}{ Microscope } & Manufacturer & Carl Zeiss Microscopy GmbH & Sensofar Metrology \\
\hline & Model & Axio Imager.Z2 Vario + LSM 800 MAT & S neox \\
\hline \multirow[t]{3}{*}{ Location } & Laboratory & $\begin{array}{l}\text { TraCEr laboratory, MONREPOS, } \\
\text { Germany }\end{array}$ & $\begin{array}{l}\text { Anthrotopography Laboratory, New York } \\
\text { University, USA }\end{array}$ \\
\hline & Floor & -1 (basement) & 7 \\
\hline & Anti-vibration table & Passive & Active \\
\hline \multirow[t]{2}{*}{ Acquisition } & Software & ZEN blue 2.3 with Shuttle\&Find module & SensoSCAN 6.4 \\
\hline & Mode & LSM (laser scanning confocal microscopy) & Confocal \\
\hline \multirow[t]{3}{*}{ Objective } & Manufacturer & Carl Zeiss Microscopy GmbH & Nikon Corporation \\
\hline & Coordinate system & $\begin{array}{l}\text { C Epiplan-Apochromat } \\
10 \times / \mathrm{NA}=0.40 / \mathrm{WD}=5.4 \mathrm{~mm} \\
\mathrm{FOV}=850.8 \times 709.9 \mu \mathrm{m}\end{array}$ & $\begin{array}{l}\text { TU Plan Fluor EPI P } \\
10 \times / \mathrm{NA}=0.30 / \mathrm{WD}=17.5 \mathrm{~mm} \\
\text { FOV }=1754 \times 1320 \mu \mathrm{m}\end{array}$ \\
\hline & Surface topography & $\begin{array}{l}\text { C Epiplan-Apochromat } \\
50 \times / \mathrm{NA}=0.95 / \mathrm{WD}=0.22 \mathrm{~mm}\end{array}$ & $\begin{array}{l}\text { TU Plan Fluor EPI P } \\
50 \times / \mathrm{NA}=0.30 / \mathrm{WD}=2 \mathrm{~mm}\end{array}$ \\
\hline \multirow[t]{3}{*}{ Illumination } & Source & Laser & LED \\
\hline & Wavelength & $405 \mathrm{~nm}$ & $530 \mathrm{~nm}$ \\
\hline & Intensity & $4 \%$ & $55.50 \%$ \\
\hline \multirow[t]{5}{*}{ Settings } & Scanning direction & Both ways (no correction, line step $=1$ ) & na \\
\hline & Scanning speed & $8(\max )$ & $\times 1$ \\
\hline & Bit depth & 16 bits & 8 bits \\
\hline & Master Gain & $245 \mathrm{~V}$ & na \\
\hline & Pinhole diameter & $54 \mu \mathrm{m}$ (1 AU lateral optical resolution) & na \\
\hline \multirow{6}{*}{$\begin{array}{l}\text { Size and resolution (surface } \\
\text { topography, } 50 \times \text { ) }\end{array}$} & Zoom & $\times 0.5$ & na \\
\hline & FOV & $255.56 \times 255.56 \mu \mathrm{m}$ & $350.88 \times 264.19 \mu \mathrm{m}$ \\
\hline & Frame size & $991 \times 991$ pixels & $1360 \times 1024$ pixels \\
\hline & $\mathrm{X} / \mathrm{Y}$ pixel size & $0.258 \mu \mathrm{m}$ & $0.258 \mu \mathrm{m}$ \\
\hline & Step size & $0.25 \mu \mathrm{m}$ & $0.20 \mu \mathrm{m}$ \\
\hline & Data quality & $\begin{array}{l}\text { No noise cut }(0-65,335 \text { levels, } \\
\text { post-processing) }\end{array}$ & Sensitivity $=1.00$ \\
\hline \multirow[t]{4}{*}{ Measurement conditions } & Duration & $\sim 2-3 \mathrm{~min}$ & $\sim 1 \mathrm{~min}$ \\
\hline & $\begin{array}{l}\text { Vertical }(z) \text { measuring } \\
\text { range }\end{array}$ & $28-35 \mu \mathrm{m}$ & $60 \mu \mathrm{m}$ \\
\hline & Temperature & 22.8 to $23.8 \pm 0.5^{\circ} \mathrm{C}$ & 21.7 to $22.7 \pm 0.5^{\circ} \mathrm{C}$ \\
\hline & Relative humidity & 54.40 to $62.60 \pm 3 \% \mathrm{rH}$ & 56.90 to $62.90 \pm 3 \% \mathrm{rH}$ \\
\hline
\end{tabular}

$A U$ Airy Unit (1 AU = 1.22 $\lambda / \mathrm{NA}), F O V$ field of view, $n a$ not applicable, $N A$ numerical aperture, $W D$ working distance

Much more work is needed to define the best way to analyze the surfaces of archeological tools but this task is beyond the scope of the present study. The processing workflow was performed consistently to enable the comparison, which was the goal. It is not intended as a general recommendation on how to measure surfaces of experimental or archeological samples.

On each thresholded S-L surface, we first manually measured, with the "parallel lines" distance measurement study, the $X$ and $Y$ distances from an easily identifiable feature to the sides of the image in full screen view (Online Resource 3). This tool cannot constrain the measurement to either $X$ or $Y$ directions, but we adjusted the lines so that the $X$ and $Y$ angles deviated less than $0.1^{\circ}$ from pure $X / Y$ directions, i.e., $90^{\circ}$ and $0^{\circ}$ ( $X$ direction) or $0^{\circ}$ and $-90^{\circ}$ ( $Y$ direction).

Additionally, in order to have an idea of the texture variation between the repeated measurements, ISO 25178-2 roughness parameters (Online Resource 4) were computed from the thresholded S-L surfaces (Online Resource 5).

\section{Statistical procedure}

As explained above, a comparison of positional accuracies between the microscopes was not the goal of this study. The statistical analyses therefore focus on measuring the positional repeatability of each microscope individually, and how 
Table 2 Descriptive statistics of $X / Y$ shifts on both microscopes

\begin{tabular}{|c|c|c|c|c|c|c|c|c|c|c|c|}
\hline \multirow[b]{2}{*}{ Microscope } & \multirow[b]{2}{*}{$n$} & \multicolumn{5}{|c|}{$X(\mu \mathrm{m})$} & \multicolumn{5}{|c|}{$Y(\mu \mathrm{m})$} \\
\hline & & Min & Max & Mean & Median & SD & Min & Max & Mean & Median & SD \\
\hline LSM & 9 & 18.85 & 88.73 & 41.84 & 33.30 & 20.39 & 1.57 & 52.11 & 23.57 & 18.07 & 17.02 \\
\hline S neox & 9 & 5.01 & 129.30 & 58.46 & 50.23 & 41.44 & 0.79 & 61.37 & 28.09 & 27.10 & 20.39 \\
\hline
\end{tabular}

$n$ sample size, min minimum, $\max$ maximum, $S D$ standard deviation

variable the results of topographic analyses are, due to this positional (in)accuracy.

The absolute value of the difference between the $X / Y$ distances from each scan and the $X / Y$ distances from the first scan was calculated. This gives a measure of the shift in $X / Y$ directions between the repeated measurements (Online Resource 5). The minimum, maximum, mean, median, and standard deviation of these $X / Y$ shifts were calculated for each microscope $(n=2 \times 9)$.

The same statistics were also calculated for each ISO 25178-2 parameter, for each microscope, on all scans $(n=$ $2 \times 10)$.

All statistical analyses were performed in the open-source software R (v. 3.5.2; R Core Team 2018) through RStudio (v. 1.1.463; RStudio Inc., Boston, USA) for Microsoft Windows 10. The following packages were used: doBy (v. 6.4-2; Højsgaard and Halekoh 2018), ggplot2 (v. 3.1.0; Wickham 2016), openxlsx (v. 4.1.0; Walker 2018), R.utils (v. 2.7.0; Bengtsson 2018). Reports of the analyses in HTML format, created with the knitr (v. 1.21; Xie 2014, 2015, 2018) and rmarkdown (v. 1.11; Allaire et al. 2018) packages for $\mathrm{R} / \mathrm{RStudio}$, are available as Online Resource 6.

\section{Results}

\section{Shift $X / Y$}

The mean shift is larger in $X$ than $Y$ direction for both microscopes (Table 2, Fig. 3, Online Resource 5, and script no. 3 of Online Resource 6): around 42 and $58 \mu \mathrm{m}(X)$, and around 24 and $28 \mu \mathrm{m}(Y)$, for the LSM and S neox respectively. The same trend can be observed on the medians. The positional repeatability varies greatly around these central tendencies: from less than $1 \mu \mathrm{m}$ ( $Y$ direction on the $\mathrm{S}$ neox) to more than $129 \mu \mathrm{m}$ ( $X$ direction on the $\mathrm{S}$ neox).

The $X$ and $Y$ shifts, as well as their variations, are smaller on the LSM than on the S neox.

\section{D surface texture}

Six parameters, spanning the different categories of field parameters (Online Resource 4), are plotted on Fig. 4; plots for the other 23 parameters can be found in the HTML output for the statistical analysis (script no. 3 of Online Resource 6). The $S a$ and $S q$ parameters are different measures of surface roughness (Blateyron 2013). Sxp is the height difference between the average height of the surface ( $p=50 \%$ material ratio) and the highest peak, excluding the $2.5 \%$ highest points $(q=$ 97.5\% material ratio). Str is a measure of isotropy; it varies between 0 (anisotropic surface) and 1 (isotropic surface). Std calculates the main direction of the surface, but is obviously only relevant for anisotropic surfaces, which is not the case here (Str > 0.6). Vmc is the volume of material (i.e., below the surface), excluding the $10 \%$ lowest $(p=10 \%)$ and $20 \%$ highest $(q=80 \%)$ points.

The variation in the measurement of surface texture between the repeated measurements is substantial for most parameters (Fig. 4; Online Resources 5-7). In other words, all quantified properties of the surface texture (height, roughness, isotropy, volume...; Online Resource 4) are impacted by the $X$ -

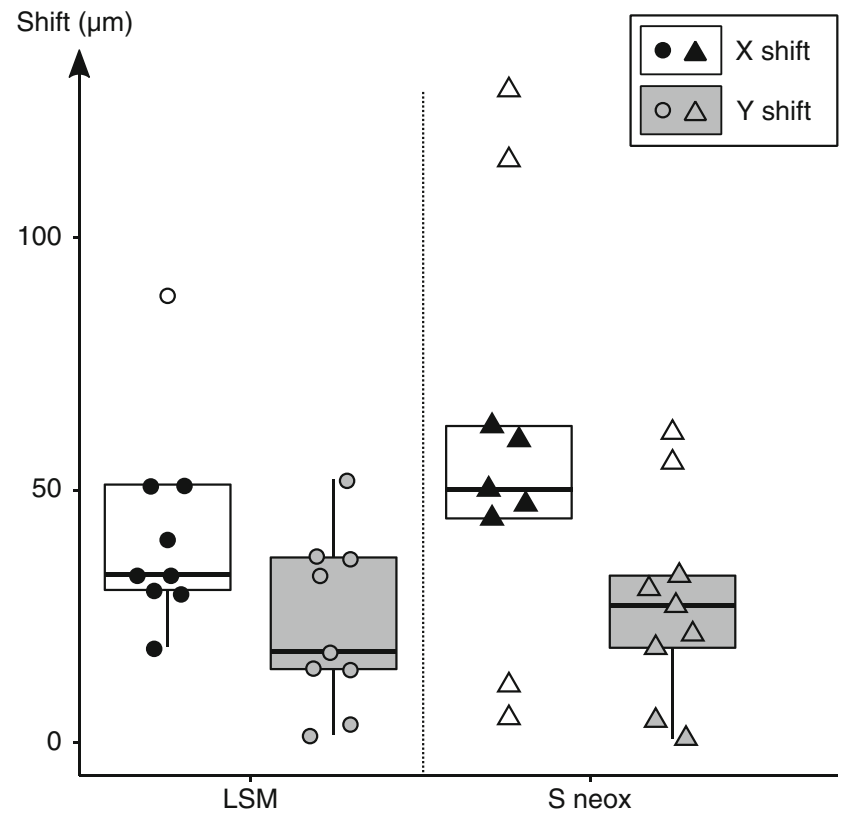

Fig. 3 Boxplots of $X / Y$ shifts on both microscopes. The boxes represent the interquartile range (IQR), i.e., between the 25th and 75th percentile, with the median shown as a thick horizontal line. The bars extend up to 1.5 IQR on each side of the box. Empty symbols represent outliers (points beyond 1.5 IQR). The points are spread horizontally within each group so that none are superimposed for readability 
Fig. 4 Boxplots of selected ISO 25178 parameters: $S a, S q, S x p$, Str, Std, and Vmc. See Fig. 3 for details on boxplots and symbols
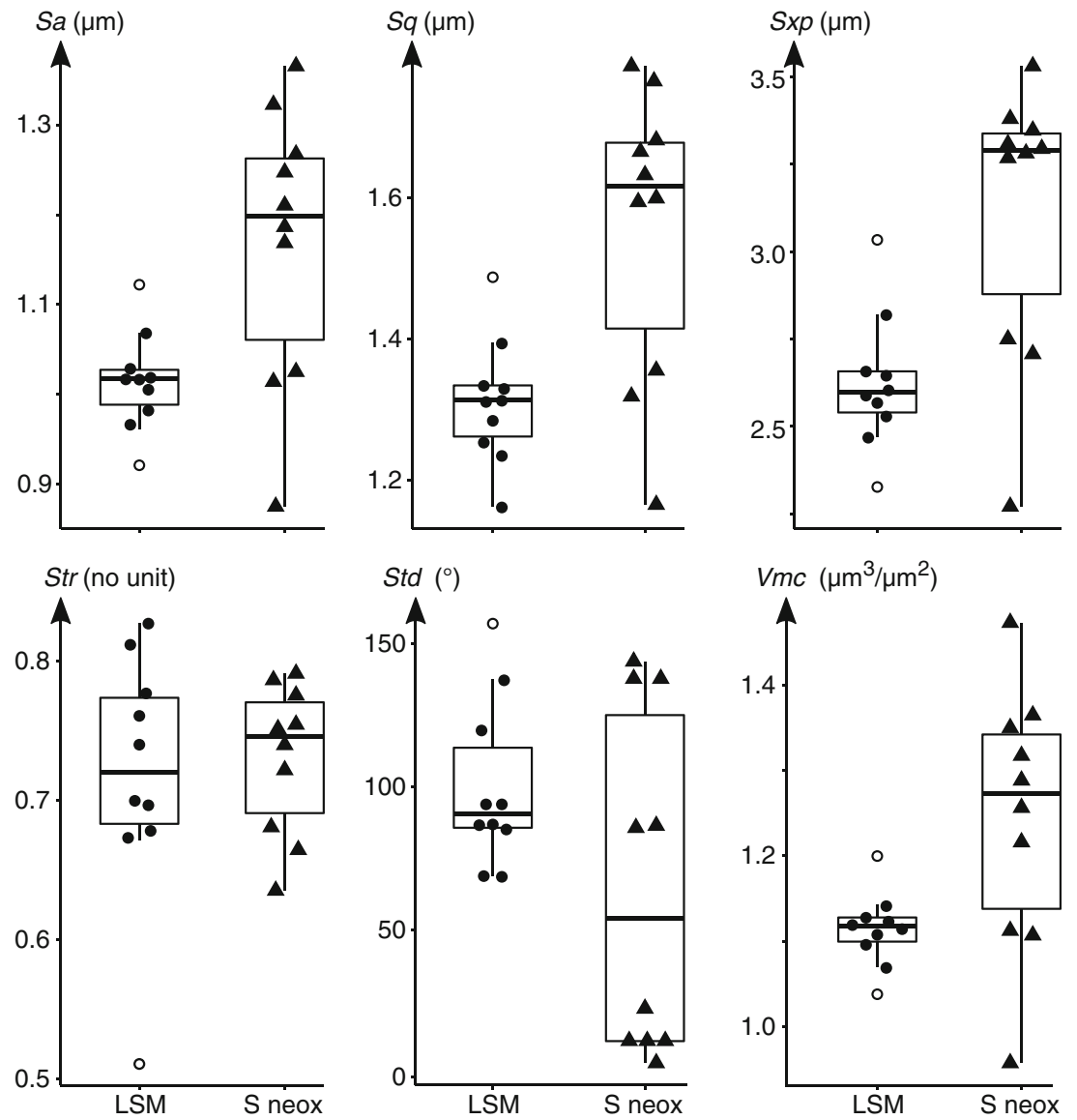

$Y$ shift from the coordinate system functionality. The variability between the measurements is larger on the $\mathrm{S}$ neox than on the LSM for 20 out of 29 ISO 25178 parameters, spanning all categories except height parameters and probably resulting from the larger $X-Y$ shift.

\section{Discussion}

The proposed coordinate system functionality is articulated into two main steps. First, three ceramic beads are adhered onto the sample's surface to serve as reference markers. In case of experimental samples, epoxy resin is preferred so that the beads are permanently and strongly adhered. It is indeed important that the beads are not removed during the experiments, as the coordinate system cannot be recreated if one or more beads get detached or displaced. For archeological samples, Paraloid B72 can be used because it is easily reversible. However, this is meaningful only if the beads can be adhered for at least two sequential imaging acquisitions before being removed.

Second, the software packages of the confocal microscopes use these markers to define a coordinate system on the sample. Every scan is then relative to this coordinate system and it is therefore possible to automatically relocate the position.

\section{Comparison of software packages}

The same experimental stone tool was scanned with two confocal microscopes: a Zeiss LSM 800 MAT operated with the ZEN blue software package (together with the Shuttle-andFind module), and a Sensofar S neox with SensoSCAN software. Both software packages offer a coordinate system functionality but the workflow is different and most variations result from a different original application. This part of the Sensofar software was developed to be used as a template, i.e., to prepare a routine to analyze different samples in identical ways. The Shuttle-and-Find module of Zeiss was developed for correlative microscopy, i.e., for imaging the same spot on a given sample with different pieces of equipment (e.g., light and scanning electron microscopes [SEM]). This is why SensoSCAN needs a template for the acquisition parameters ("single measurement recipe", SMR), while the acquisition settings can be adjusted for each measurement and the coordinate system is calibrated before acquisition in ZEN blue. The SensoSCAN workflow could become problematic if the sample or its orientation has changed significantly, so that the $z$-range is too small for the new acquisition. In this case, 
the $z$-range should be increased in advance in the SMR to account for this possibility; this is why we set it to $60 \mu \mathrm{m}$ (Table 1). The very fast acquisition of the $\mathrm{S}$ neox, as compared to the LSM, makes this solution practical. Additionally, each position can be assigned a different SMR, so that each position can be acquired with appropriate settings. On the other hand, while it is possible to plan several acquisitions on the same sample in ZEN blue (with another optional module), each acquisition must be run with the same settings.

Some other differences are noteworthy. The size of the crosshair is fixed in ZEN blue while the diameter of the circle can be adjusted to fit the diameter of beads in SensoSCAN, which should improve the positional repeatability, although the $X-Y$ deviation was observed to be higher for the $\mathrm{S}$ neox (Table 2). The Shuttle-and-Find module (Zeiss) also offers more possibilities: the coordinate system can be (1) mirrored so that the same system can be applied to originals, molds, and casts, and (2) transferred between different microscopes (light, digital, and confocal microscopes, and SEM) as long as each of them features a motorized XY stage and the Shuttle-andFind module. Finally, in SensoSCAN, the stage moves automatically to where it expects the next reference marker. We did not find an option to deactivate this behavior that could be dangerous for some samples and the objectives, as the objective could then crash into the sample. This behavior can be turned off in ZEN blue (Online Resource 2, step 6a).

\section{Accuracy of the method}

The positional repeatability of the coordinate system functionality depends on several factors: positional repeatability of the motors of the XY stage, rounding errors of the algorithms, and transformations unaccounted for (rotation and tilt, see below). The mean absolute positional repeatability (combined mean $X$ and $Y$ shifts via Pythagoras' formula), taking into account all these factors, is $48.02 \mu \mathrm{m}$ for the LSM and $64.86 \mu \mathrm{m}$ for the $\mathrm{S}$ neox. However, these values should be considered relative to the acquired field of view, larger with the $\mathrm{S}$ neox. The relative positional repeatability could be defined as the absolute repeatability divided by the field of view diagonal, resulting in a unit-less value. This gives $13.29 \%$ on the LSM and $14.77 \%$ on the S neox. Therefore, a deviation of about $14 \%$ of the field of view can be expected when using the coordinate system functionality on both microscopes. We expect that the positional repeatability of microscopes from other manufacturers will be in the same range.

Ideally, the positional repeatability should be as small (i.e., small deviation) as possible, but practical and technical constraints limit it to $14 \%$ of the field of view. While this value might seem too large, we argue it still represents an improvement over visual relocation, because (1) it is faster and can be applied on every sample routinely, (2) it does not depend on the recognition of features that might be removed through wear formation during experiments, or that might not be recognizable or look differently with different pieces of equipment, and (3) after the approximate position is found again with the functionality, fine visual adjustments could still be performed quickly to improve the relocation.

To our knowledge, there are only three studies that applied ISO 25178 parameters $(S q)$ to a set of experimental flints used on different worked materials. Evans and Macdonald (2011) used their experimental tools on antler, dry hide, fresh hide, greasy hide, and wood and acquired their surface topography with a laser-scanning confocal microscope (LSCM). Their Fig. $3 \mathrm{~b}$ shows the means \pm 2 standard deviations for each worked material, calculated from 3.8 and $10.1 \mu \mathrm{m}$ areas extracted from the acquired surfaces. The doubled standard deviation of $S q$ from the LSM (Online Resource 7) would not blur the differences on the large areas between the worked materials. The measured variation of $S q$ on the $\mathrm{S}$ neox is larger, so that the different worked materials would be indistinguishable; the difference between unused and used samples would also vanish. Macdonald (2014) used experimental flints on antler, wood, hide, meat, and wheat and imaged their surfaces with focus variation microscopy. Based on her Table 3, it seems that the variation in $S q$ from the LSM would likely still allow the distinction between the worked materials, while the variability from the $\mathrm{S}$ neox would probably blur the differences. Unfortunately, Ibáñez et al. (2018) published only the results of their discriminant analysis; therefore, $S q$ values cannot be compared.

However, the $S q$ values from these other studies (Evans and Macdonald 2011; Macdonald 2014) are not directly comparable to ours because the size of the analyzed areas and the processing steps are different. Moreover, these studies focused on polished areas, where $S q$ is expected to be lower, while we analyzed the whole image frame. So it remains to be tested whether the variations in ISO parameters due to the coordinate system repeatability would indeed mask potential differences between worked materials, use duration, applied forces, or any other experimental variable.

\section{Limitations and future improvements}

The application of reference markers on the sample as presented here with adhesives and ceramic beads is already easy and cheap, but it can be further improved. The ratio of epoxy resin and hardener is difficult to measure for the small quantities needed to adhere a few beads on a few samples. So there is usually a lot of waste to ensure adequate quantities for efficient mixing. An alternative would be to work with UHU Endfest epoxy resin in dual-barrel cartridges with mixing tips.

While the process worked well with epoxy resin, the Paraloid B72 proved difficult to work with because of its short curing time and, in high dilution, low viscosity, leading to larger-than-needed areas covered in glue (Fig. 1b). Adjusting 
the ratio of Paraloid B72 to ethyl acetate will surely improve the applicability of this glue, and therefore the applicability of this process to archeological samples.

The orientation of a sample on a microscope XY stage can vary along three geometric transformations: translation (linear movement in the horizontal XY plane), rotation (circular movement in the horizontal XY plane), and tilt (inclination relative to the horizontal XY plane). The software packages used here can mathematically correct for translation of the sample on the stage: it does not matter where the sample is located on the stage (Fig. 5a). However, rotation on the stage is only partially accounted for: the center of the field of view is located on the same point of the sample, but the field of view is rotated, meaning that different parts of the sample are imaged (Fig. 5b). Finally, tilt cannot be fully corrected because the size of the imaged part of the sample varies with tilt (Fig. 5c). Furthermore, the software packages used here do not correct for tilt but account only for horizontal transformations (translation and rotation). The positional repeatability of the coordinate system therefore depends greatly on the precision of the re-positioning (rotation and tilt) on the stage. A molded support can be used to reproducibly orientate (tilt) the sample on the microscope stage (similar to Fig. 2). Alternatively, or additionally, a two-axis goniometer and/or a rotational stage with micrometer screws would allow the precise reorientation of the sample.

As a final note, defining the area of interest before running the experiment as shown here is not without its problems. Most usewear studies analyze areas showing polish (i.e., shiny, flattened surfaces in light microscopy; e.g., Marreiros et al. 2015 and references therein) because the polished areas are assumed to represent the most distinctive wear. However, the extent of these polished areas depends on many factors (among others, duration of use, force applied, and worked material; Marreiros et al. 2015 and references therein). This implies that it is not possible to know before the experiment where polish is going to develop on the sample. Three strategies can be employed to deal with this problem, in order to be able to compare the surface topography before, during, and after an experiment. First, a sufficient number of areas can be analyzed before the experiment so that at least one will show use-wear at the end of the experiment; this is likely not an efficient approach. Second, algorithms could be used to automatically extract and analyze the polished areas only within a large enough acquired surface, as Evans et al. (2014) did, with partial success. Finally, visual identification of polish probably represents the end stage of a continuous wear process (e.g., Grace 1989); earlier wear stages, where polish cannot be observed with light microscopy or SEM, could still be quantitatively distinctive of different uses (e.g., worked material, force, duration).

\section{Conclusions}

In this study, we presented a simple protocol to create a coordinate system on a sample that can be used to automatically relocate a given position on such sample. The workflow can be split into three main steps: (1) adhere three ceramic beads onto the sample that will serve as reference markers, (2) set up the coordinate system and define the position(s) of interest on the sample, and (3) use the coordinate system functionality to automatically relocate these position(s).

We first demonstrated the applicability of this approach on an experimental flint blade by adhering beads with epoxy resin onto the sample. We also showed that the beads can be attached to archeological samples if Paraloid B72 is used, because this polymer is reversible with solvents such as acetone or ethyl acetate. We then applied and tested this method with two confocal microscopes: a laser-scanning confocal microscope manufactured by Carl Zeiss Microscopy GmbH (LSM 800 MAT) and an optical profilometer by Sensofar Metrology (S neox). This method can be used with any microscope with a motorized XY stage and the same software functionalities: all types of microscopes from Zeiss; all profilometers from Sensofar (S neox and its predecessors) and Leica (DCM8); the Olympus LEXT presumably has a similar functionality, too. Positional repeatability is likely to vary depending on the equipment due to the motors' repeatability and software algorithms.

As all methods, this method has limitations and the relative positional repeatability of about $14 \%$ of the field of view might seem large. On the other hand, it still represents a great a

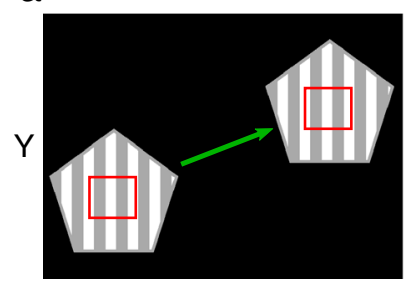

$\mathrm{X}$ $\mathrm{b}$

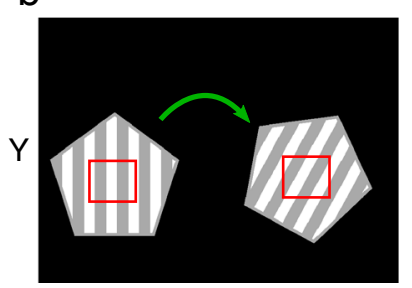

$\mathrm{X}$
C

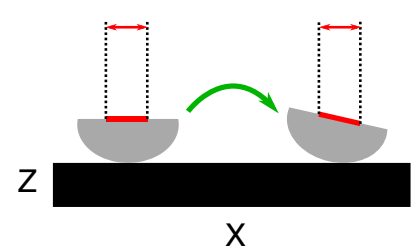

Fig. 5 Schematic drawing of translation (a), rotation (b), and tilt (c). A component of translation is included in $\mathbf{b}$ and $\mathbf{c}$ as well, for readability. Black $=$ microscope stage, red $=$ field of view, green arrows $=$ transformation, gray stripped pentagon $=$ sample, $X-Y-Z=$ microscope directions ( $X$ and $Y$ axes define the horizontal plane, and $Z$ is the vertical axis) 
improvement over visual relocation, especially for quantitative use-wear analyses. In some cases, it can be used as a first step. Fine adjustments could be made visually so that the position(s) can be relocated more quickly. Alternatively, the "4D Series Analysis" module of ConfoMap/MountainsMap could be used to automatically perform the fine alignments. However, if the surface has changed too much (e.g., due to experimentation) or if the different pieces of equipment image the sample in ways that are difficult to compare visually (e.g., light microscope vs. SEM), this coordinate system functionality is the best option.

We regard this coordinate system functionality as an important step toward repeatability and reproducibility in experimental studies. With this method, it is not only possible and easier to analyze the same location(s) on a sample during sequential experiments, but also to re-analyze the same sample in a comparable way, either with different pieces of equipment for further analysis or by different researchers. We therefore hope that this protocol will contribute to the development of reproducible experimental programs and archeological research, especially in, but not limited to, use-wear analysis.

Acknowledgments We thank Sigmund Lindner GmbH for providing us the ceramic beads used for the coordinate system, and BTC Europe $\mathrm{GmbH}$ for the detergent used to clean the samples. We also thank Adam M. Platteis (Sensofar Metrology) and Dr. Matthias Vaupel (Carl Zeiss Microscopy $\mathrm{GmbH}$ ) for their help with the microscopes.

Funding This research has been supported within the RömischGermanisches Zentralmuseum - Leibniz Research Institute for Archeology by German Federal and Rhineland Palatinate funding (Sondertatbestand "Spurenlabor") and is publication no. 1 of the TraCEr laboratory. AR and RI thank New York University for funding the Anthrotopography Laboratory.

\section{Compliance with ethical standards}

Conflict of interest The authors declare that they have no conflict of interest.

Open Access This article is distributed under the terms of the Creative Commons Attribution 4.0 International License (http:// creativecommons.org/licenses/by/4.0/), which permits unrestricted use, distribution, and reproduction in any medium, provided you give appropriate credit to the original author(s) and the source, provide a link to the Creative Commons license, and indicate if changes were made.

Publisher's note Springer Nature remains neutral with regard to jurisdictional claims in published maps and institutional affiliations.

\section{References}

Allaire JJ, Xie Y, McPherson J et al (2018) rmarkdown: Dynamic Documents for R. R package version 1.11. https://CRAN.Rproject.org/package=rmarkdown. Accessed 27 Sep 2018
Asryan L, Ollé A, Moloney N (2014) Reality and confusion in the recognition of post-depositional alterations and use-wear: an experimental approach on basalt tools. J Lithic Stud 1:9-32. https://doi. org/10.2218/jls.v1i1.815

Bengtsson H (2018) R.utils: Various Programming Utilities. R package version 2.7.0. https://CRAN.R-project.org/package=R.utils. Accessed 27 Sep 2018

Benito-Calvo A, Arroyo A, Sánchez-Romero L, Pante M, de la Torre I (2018) Quantifying 3D micro-surface changes on experimental stones used to break bones and their implications for the analysis of early stone age pounding tools. Archaeometry 60:419-436. https://doi.org/10.1111/arcm.12325

Blateyron F (2013) The areal feature parameters. In: Leach R (ed) Characterisation of areal surface texture. Springer Berlin Heidelberg, Berlin, Heidelberg, pp 45-65

Bofill M, Procopiou H, Vargiolu R, Zahouani H (2013) Use-wear analysis of Near Eastern prehistoric Grinding stones. In: Anderson PC, Cheval C, Durand A (eds) Regards croisés sur les outils liés au travail des végétaux. An interdisciplinary focus on plant-working tools. APDCA, Antibes, pp 219-236

Chabot J, Dionne M-M, Paquin S (2017) High magnification use-wear analysis of lithic artefacts from Northeastern America: creation of an experimental database and integration of expedient tools. Quat Int 427:25-34. https://doi.org/10.1016/j.quaint.2015.11.061

Evans AA, Donahue RE (2005) The elemental chemistry of lithic microwear: an experiment. J Archaeol Sci 32:1733-1740. https:// doi.org/10.1016/j.jas.2005.06.010

Evans AA, Donahue RE (2008) Laser scanning confocal microscopy: a potential technique for the study of lithic microwear. J Archaeol Sci 35:2223-2230. https://doi.org/10.1016/j.jas.2008.02.006

Evans AA, Macdonald D (2011) Using metrology in early prehistoric stone tool research: further work and a brief instrument comparison. Scanning 33:294-303. https://doi.org/10.1002/sca.20272

Evans AA, Macdonald DA, Giusca CL, Leach RK (2014) New method development in prehistoric stone tool research: evaluating use duration and data analysis protocols. Micron 65:69-75. https://doi.org/ 10.1016/j.micron.2014.04.006

Galimova M, Sitdikov A (2017) Prehistoric flint scrapers or gun-lock flints? Experimental and use-wear criteria (according to archaeology of Kazan, Tatarstan). Quat Int 427:74-79. https://doi.org/10.1016/j. quaint.2015.12.006

Grace R (1989) Interpreting the function of stone tools: the quantification and computerisation of microwear analysis. B.A.R. Internation Series 474. Archaeopress, Oxford

Højsgaard S, Halekoh U (2018) doBy: Groupwise Statistics, LSmeans, Linear Contrasts, Utilities. R package version 4.6-2. https://CRAN. R-project.org/package=doBy. Accessed 27 Sep 2018

Ibáñez JJ, Lazuen T, González-Urquijo J (2018) Identifying experimental tool use through confocal microscopy. J Archaeol Method Theory. https://doi.org/10.1007/s10816-018-9408-9

International Organization for Standardization (1996) ISO 4288 Geometrical product specifications (GPS) - Surface texture: Profile method - Rules and procedures for the assessment of surface texture

International Organization for Standardization (1997) ISO 4287 Geometrical product specifications (GPS) - Surface texture: Profile method - Terms, definitions and surface texture parameters

International Organization for Standardization (2012) ISO 25178-2 geometrical product specifications (GPS) - surface texture: areal part 2: terms, Definitions and surface texture parameters

Key AJM, Stemp WJ, Morozov M, Proffitt T, de la Torre I (2015) Is loading a significantly influential factor in the development of lithic microwear? An experimental test using LSCM on basalt from Olduvai Gorge. J Archaeol Method Theory 22:1193-1214. https:// doi.org/10.1007/s10816-014-9224-9 
Leach R (ed) (2013) Characterisation of areal surface texture. SpringerVerlag, Berlin Heidelberg

Macdonald DA (2014) The application of focus variation microscopy for lithic use-wear quantification. J Archaeol Sci 48:26-33. https://doi. org/10.1016/j.jas.2013.10.003

Marreiros JM, Mazzucco N, Gibaja JF, Bicho N (2015) Macro and micro evidences from the past: the state of the art of archeological use-wear studies. In: Marreiros JM, Gibaja Bao JF, Ferreira Bicho N (eds) Use-wear and residue analysis in archaeology. Springer International Publishing, Cham, pp 5-26

Martisius NL, Sidéra I, Grote MN, Steele TE, McPherron SP, SchulzKornas E (2018) Time wears on: assessing how bone wears using 3D surface texture analysis. PLoS One 13:e0206078. https://doi.org/ 10.1371/journal.pone. 0206078

Ollé A, Vergès JM (2014) The use of sequential experiments and SEM in documenting stone tool microwear. J Archaeol Sci 48:60-72. https:// doi.org/10.1016/j.jas.2013.10.028

Pedergnana A, Asryan L, Fernández-Marchena JL, Ollé A (2016) Modern contaminants affecting microscopic residue analysis on stone tools: a word of caution. Micron 86:1-21. https://doi.org/10. 1016/j.micron.2016.04.003

Portillo M, Bofill M, Molist M, Albert RM (2013) Phytolith and use-wear functional evidence for grinding stones from the Near East. In: Anderson PC, Cheval C, Durand A (eds) Regards croisés sur les outils liés au travail des végétaux. An interdisciplinary focus on plant-working tools. APDCA, Antibes, pp 205-218

Queffelec A, Rosso D, d'Errico F (2018) The use of ochre 40,000 years ago in Africa. Sensofar Metrology, Barcelona

R Core Team (2018) R: a language and environment for statistical computing. R Foundation for Statistical Computing, Vienna, Austria. Version 3.5.2. https://www.R-project.org/. Accessed 27 Sep 2018

Sano K, Oba M (2015) Backed point experiments for identifying mechanically-delivered armatures. J Archaeol Sci 63:13-23. https://doi.org/10.1016/j.jas.2015.08.005

Stemp WJ, Stemp M (2001) UBM laser profilometry and lithic use-wear analysis: a variable length scale investigation of surface topography. J Archaeol Sci 28:81-88. https://doi.org/10.1006/jasc.2000.0547

Stemp WJ, Stemp M (2003) Documenting stages of polish development on experimental stone tools: surface characterization by fractal geometry using UBM laser profilometry. J Archaeol Sci 30:287296. https://doi.org/10.1006/jasc.2002.0837

Stemp WJ, Lerner HJ, Kristant EH (2013) Quantifying microwear on experimental Mistassini quartzite scrapers: preliminary results of exploratory research using LSCM and scale-sensitive fractal analysis. Scanning 35:28-39. https://doi.org/10.1002/sca.21032

Stemp WJ, Morozov M, Key AJM (2015) Quantifying lithic microwear with load variation on experimental basalt flakes using LSCM and area-scale fractal complexity (Asfc). Surf Topogr Metrol Prop 3: 034006. https://doi.org/10.1088/2051-672X/3/3/034006

Stemp WJ, Lerner HJ, Kristant EH (2018) Testing area-scale fractal complexity (Asfc) and laser scanning confocal microscopy (LSCM) to document and discriminate microwear on experimental quartzite scrapers. Archaeometry 60:660-677. https://doi.org/10.1111/arcm. 12335

Walker A (2018) openxlsx: Read, Write and Edit XLSX Files. R package version 4.1.0. https://CRAN.R-project.org/package=openxlsx. Accessed 27 Sep 2018

Watson AS, Gleason MA (2016) A comparative assessment of texture analysis techniques applied to bone tool use-wear. Surf Topogr Metrol Prop 4:024002. https://doi.org/10.1088/2051-672X/4/2/ 024002

Werner JJ (2018) An experimental investigation of the effects of postdepositional damage on current quantitative use-wear methods. J Archaeol Sci Rep 17:597-604. https://doi.org/10.1016/j.jasrep. 2017.12.008

Wickham H (2016) ggplot2: Elegant Graphics for Data Analysis. Springer, New York

Xie Y (2014) knitr: A Comprehensive Tool for Reproducible Research in R. In: Stodden V, Leisch F, Peng RD (eds) Implementing Reproducible Computational Research. Chapman and Hall/CRC, Boca Raton

Xie Y (2015) Dynamic Documents with R and knitr, 2nd edn. Chapman and Hall/CRC, Boca Raton

Xie Y (2018) knitr: A General-Purpose Package for Dynamic Report Generation in R. R package version 1.21. https://yihui.name/knitr/. Accessed 27 Sep 2018 